\title{
Cálculo teórico de propriedades estruturais e energéticas de grafeno e óxido de grafeno
}

Beatriz C. Guedes ${ }^{1 *}(\mathrm{IC})$, José R. S. Politi ${ }^{1}(\mathrm{PG})$. beatrizcostagguedes@gmail.com.

${ }^{1}$ Laboratório de Química Computacional, Instituto de Química, Universidade de Brasília, Brasília/Distrito Federal.

Grafeno, DFT, correções de van der Waals, análise estrutural do óxido de grafeno.

O grafeno é uma forma alotrópica do carbono, sendo que é formado por uma camada bidimensional de carbonos ligados entre si com hibridização $\mathrm{sp}^{2}$. [1] Quando há grupos funcionais oxigenados na estrutura, tais como carbonilas, epóxidos, alcoóis e ácidos carboxílicos, têm-se o óxido de grafeno. Tanto o grafeno quanto o óxido de grafeno apresentam propriedades físico-químicas peculiares que os habilitam para diversas aplicações, tais como: placas fotovoltaicas, sensores eletroquímicos e capacitores de baterias. [2] Diante dessas aplicações, o objetivo do trabalho é descrever a geometria e a estrutura eletrônica desses dois materiais por meio da Teoria do Funcional Densidade (DFT).

Foi utilizado o modelo periódico para descrever os sistemas com o programa VASP com os funcionais: PBE, Perdew-Burke-Ernzenrhof, com aproximação GGA, Generalized Gradiente Aproximation, PBE com correção de van der Waals D3-BJ, vdW-DF, optPBE-vdW, optB86b-vdW e vdW-DF2.

Primeiramente foi estudado o tamanho da célula unitária, sendo importante observar a concordância entre os dados experimentais em relação ao custo computacional. Depois de definido o tamanho adequado da célula unitária, estudou-se apenas uma folha de grafeno com todos funcionais selecionados.Em seguida foi estudada a interação de duas folhas de grafeno, empregando os funcionais que melhor descreveram as propriedades de uma folha só. Para esse estudo foram utilizados dois modelos. [3]

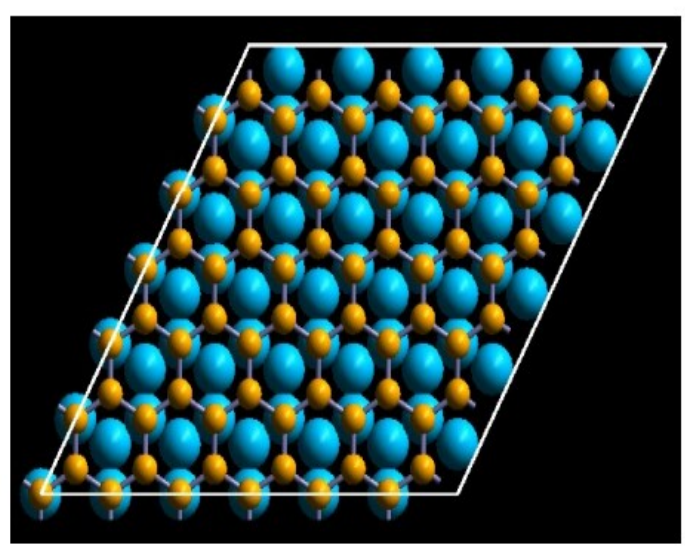

Estrutura com uma das folhas deslocada em relação á outra.

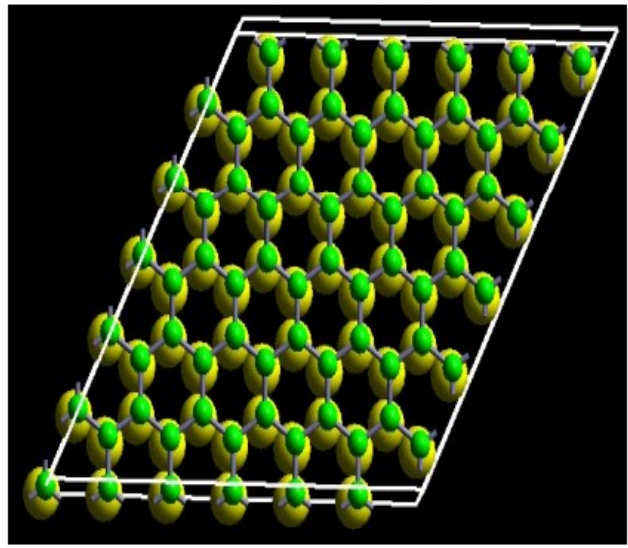

Estrutura com as duas folhas sobrepostas.

Figura 1 - Dois modelos estudados com o sistema composto de duas folhas de grafeno. 
DOI 10.21826/9788563800374026

\begin{tabular}{|c|c|c|c|c|}
\hline & $\begin{array}{c}\text { Distância entre } \\
\text { as folhas de } \\
\text { grafeno } \\
\text { sobrepostas }\end{array}$ & $\begin{array}{c}\text { Energia de } \\
\text { interação entre as } \\
\text { folhas de grafeno } \\
\text { sobrepostas }\end{array}$ & $\begin{array}{c}\text { Distância entre } \\
\text { as folhas de } \\
\text { grafeo } \\
\text { deslocadas }\end{array}$ & $\begin{array}{c}\text { Energia de } \\
\text { interação entre as } \\
\text { folhas de grafeno } \\
\text { deslocadas }\end{array}$ \\
\hline PBE & $3,6 \AA$ & $-2,71 \mathrm{eV}$ & $3,2 \AA$ & $-3,6 \mathrm{eV}$ \\
\hline PBE+D3-BJ & $3,6 \AA$ & $-2,88 \mathrm{eV}$ & $3,4 \AA$ & $-3,43 \mathrm{eV}$ \\
\hline opt-PBE-vdW & $5 \AA$ & $-1,54 \mathrm{eV}$ & $6,48 \AA$ & $-0,41 \mathrm{eV}$ \\
\hline optB86b-vdW & $4,99 \AA$ & $-1,42 \mathrm{eV}$ & $6,27 \AA$ & $-0,46 \mathrm{eV}$ \\
\hline vdW-DF & $4,98 \AA$ & $-1,47 \mathrm{eV}$ & $6,83 \AA$ & $-0,28 \mathrm{eV}$ \\
\hline vdW-DF2 & $5 \AA$ & $-1,04 \mathrm{eV}$ & $6,21 \AA$ & $-0,27 \mathrm{eV}$ \\
\hline optB88-vdW & $5,03 \AA$ & $-1,33 \mathrm{eV}$ & $7,79 \AA$ & $-0,26 \mathrm{eV}$ \\
\hline
\end{tabular}

Figura 2 - Distância e energia de interação entre as folhas de grafeno com os dois modelos estudados.

Com base nos resultados obtidos para as duas folhas de grafeno, foram escolhidos os seguintes funcionais para serem utilizados nos cálculos referentes ao óxido de grafeno: PBE com a aproximação GGA, PBE com a correção D3-BJ, optPBE-vdW e optB86b-vdW.

Inicialmente, para o óxido de grafeno, foram estudados quais são os grupos funcionais oxigenados predominantes na estrutura, analisando individualmente como cada grupo interage com a folha de grafeno, os grupos funcionais analisados foram: carbonilas, álcool e ácido carboxílico.

Através da análise dos resultados obtidos, podemos afirmar que para representar corretamente as interações entre as folhas de grafeno, é preciso utilizar funcionais que apresentem correções de van der Waals a fim de manter as folhas de grafeno no plano e para que haja interação entre as folhas. No estudo do óxido de grafeno foram utilizados os mesmos funcionais usados nas duas folhas de grafeno e que apresentavam correções de van der Waals.

\section{Agradecimentos}

Ao CNPq, á FAP-DF e a Universidade de Brasília pelo financiamento e estrutura que permitiram o desenvolvimento desse trabalho.

\section{Referências}

[1] Geim, A.K.; Novoselov, K.S. The rise of graphene. Nature Mater., 2007, 6(3), 183-191.

[2] Beatriz A. Vessali; Cecilia A. Zito; Tarcísio M. Perfecto; Diogo P.Volanti; Talita Manzon; $\mathrm{ZnO}$ nanorods/graphene oxide sheets prepared by chemical bath deposition for volatile organic compounds detection; Journal of Alloys and Compounds 696 (2017) 996-1003.

[3] A. S. Dobrota, I. A. Pasti; A Review of Theoretical Studies on Functionalized Graphene for Eletrochemical Energy Conversion and Storage Applications ;Current Physical Chemistry 2017, 6 , 244-265. 